\title{
Utility of circulating tumor cells in stage II colorectal cancer patients undergoing curative resection
}

\author{
Jun-Hui Yu ${ }^{1,2}$, Dong Wang ${ }^{1}$, Lan Jin ${ }^{1}$, Jin Wang ${ }^{1}$, Xiao-Mu Zhao ${ }^{1}$, Guo-Cong Wu ${ }^{1}$, Hong-Wei Yao ${ }^{1}$, \\ Ying-Chi Yang ${ }^{1}$, Zhong-Tao Zhang ${ }^{1}$ \\ ${ }^{1}$ Department of General Surgery, Beijing Friendship Hospital, Capital Medical University, National Clinical Research Center of Digestive Disease, \\ Beijing 100050, China; ${ }^{2}$ Department of Peritoneal Cancer Surgery, Beijing Shijitan Hospital, Capital Medical University, Beijing 100038, China \\ Contributions: (I) Conception and design: ZT Zhang, YC Yang; (II) Administrative support: GC Wu; (III) Provision of study materials or patients: \\ D Wang, HW Yao; (IV) Collection and assembly data: JH Yu, L Jin, J Wang; (V) Data analysis and interpretation: JH Yu, XM Zhao; (VI) Manuscript \\ writing: All authors; (VII) Final approval of manuscript: All authors. \\ Correspondence to: Zhong-Tao Zhang, MD, PhD; Ying-Chi Yang, MD, PhD. Department of General Surgery, Beijing Friendship Hospital, Capital \\ Medical University, National Clinical Research Center of Digestive Disease, No. 95, Yong-An Road, Xi-Cheng District, Beijing 100050, China. \\ Email: zhangzht@ccmu.edu.cn; yangyingchi@ccmu.edu.cn.
}

\begin{abstract}
Background: The aim of this study was to determine whether circulating tumor cells (CTCs) have utility as a prognostic biomarker in stage II colorectal cancer (CRC), as well as a biomarker for the selection of patients for adjuvant chemotherapy.

Methods: CTCs were detected in peripheral blood samples obtained from 73 stage II CRC patients, using a negative enrichment and immune-fluorescence in situ hybridization (imFISH) staining method. The follow-up time ranged from 3.5 to 35.9 months, and the clinic-pathologic characteristics and recurrence free survival (RFS) were collected and analyzed.

Results: Seventy-three stage II CRC patients were included in this study. The positive rate of CTCs was $65.8 \%$ in all patients, $87.5 \%$ in recurrent patients and $59.6 \%$ in no recurrence patients. The mean RFS was 30.6 months for all patients, 28.7 months for CTC-positive patients and 34.0 months for CTC-negative patients $(\mathrm{P}=0.043)$. The mean RFS of CTC-positive and CTC-negative patients with adjuvant chemotherapy were not reached, and those without adjuvant chemotherapy were 27.7 and 33.4 months, respectively.

Conclusions: The level of CTCs may be an effective prognostic factor to predict RFS in stage II CRC patients, and has potential in selecting stage II CRC patients for adjuvant chemotherapy.
\end{abstract}

Keywords: Adjuvant chemotherapy; colorectal cancer (CRC); circulating tumor cells (CTCs); prognostic factor; stage II

Submitted Sep 01, 2019. Accepted for publication Dec 13, 2019.

doi: $10.21037 /$ tcr.2020.01.37

View this article at: http://dx.doi.org/10.21037/tcr.2020.01.37

\section{Introduction}

CRC has been ranked as the fourth most common malignancies among women and the fifth among men in China (1). Radical resection is now the standard treatment for CRC patients, and adjuvant chemotherapy is alternative depending on pathologic Tumor Node Metastasis (TNM) stage.

It is well acknowledged that adjuvant chemotherapy provides no benefit in stage I CRC patients, and a minimal benefit in stage II CRC patients. However, even after undergoing radical surgery, approximately $10 \%$ of stage I and $20 \%$ of stage II CRC patients still suffer recurrence or metastasis within 5 years (2). Considering "overtreatment" in major patients and "undertreatment" in a small subgroup of patients, the use of adjuvant chemotherapy in stage II CRC patients remains controversial (2). Meanwhile, the role of traditional clinicopathologic factors in selecting 
these patients is quite limited (3). Thus, there is an urgent need to identify an effective marker for selecting patients with additional treatment.

Circulating tumor cells (CTCs), broadly defined as tumor- or metastasis-derived cells, have been used for prognostic prediction and treatment evaluation. In highrisk CRC patients, those with positive CTCs following completion of adjuvant chemotherapy develop a higher relapse rate $(4,5)$. Such CTCs are responsible for disease relapse in these patients. Moreover, CTCs in peripheral blood at the time of diagnosis or after resection of the primary tumor may identify those early-stage patients who are at risk of developing recurrent or metastatic disease and who would benefit from adjuvant therapy (6). However, the utility of CTCs in selecting early-patients for adjuvant chemotherapy remains controversial.

In this study, we collected blood samples from 73 stage II CRC patients, and detected CTCs using a negative enrichment and immune-fluorescence in situ hybridization (imFISH) staining method, aiming to clarify the utility of CTCs in stage II CRC patients treated with curative surgery.

\section{Methods}

\section{Patients}

From March 2015 to December 2017, a total of 73 consecutive stage II CRC patients were enrolled in this study, and all patients underwent curative surgery at Beijing Friendship Hospital. The diagnosis of CRC was confirmed by endoscopic biopsy and followed by pathology in all patients. The TNM stage of primary tumor was confirmed by post-operative pathologic examination of the resected primary tumor according to National Comprehensive Cancer Network (NCCN) guidelines (Version I, 2018) (7). Patients with other malignant disease in their medical history were excluded.

Patients were followed up every 3 months for the first 2 years and every 6 months thereafter. The median followup time was 22.1 (range 3.5-35.9) months. Postoperative surveillance was carried out every 3 months, including medical history, physical examination, and laboratory studies. Abdominal ultrasonography or computed tomography was performed every 6 months, and chest radiography and total colonoscopy were conducted once a year. The recurrence free survival (RFS) was defined as the period from surgery to recurrence or metastasis. The study design was approved by the Ethical Committee of the Beijing Friendship Hospital (Ethics number: BJFHEC/2014-086). All patients had signed informed consent before enrollment.

\section{CTCs detection}

$7.5 \mathrm{~mL}$ of peripheral blood sample was collected on the 7th postoperative day via BD Vacutainer Glass Blood Collection Tubes with Acid Citrate Dextrose (Becton, Dickinson and Company, Franklin Lakes, NJ, USA). In order to avoid contamination of epithelial cells, all samples were collected after discarding the first $2 \mathrm{~mL}$ of blood. The blood samples were centrifuged at $650 \times \mathrm{g}$ at room temperature, the upper serum layer was discarded, and the remaining cells were resuspended in a lysis buffer solution. After $8 \mathrm{~min}$ of vertical mixing $(20 \mathrm{rpm})$ that facilitated lysis of red blood cells, the samples were subjected to centrifugation at $650 \times \mathrm{g}$ at room temperature, and the residual cell pellets were resuspended in phosphate buffer solution. Then, the solutions were subsequently incubated with anti-CD 45 monoclonal antibody-coated magnetic beads for $30 \mathrm{~min}$, followed by the separation of magnetic beads using a magnetic stand (Promega, Madison, WI, USA). Afterwards, the samples were centrifuged at $800 \times \mathrm{g}$ for $3 \mathrm{~min}$. Cell pellets were spotted on glass slides, and then imFISH staining was carried out (Figure 1).

The enriched CTCs were subjected to imFISH analysis, which was performed using centromere DNA probes of chromosome 7 (green) and 8 (orange) (Abbott Molecular Diagnostics, Des Plaines, IL, USA). The immunofluorescence assay was carried out with antiCD45 (red). The glass slides were washed three times with Tris-buffered saline before mounting. Cells were mounted with mounting medium containing the nuclear dye 4',6-diamidino-2-phenylindole (DAPI) and then visualized under a fluorescence microscope. Evaluation criteria for CTCs identification included both chromosome enumeration probe (CEP) $7 / 8 \geq 3$ and CD45 negative staining pattern overlying the DAPI staining of the nucleus. CTC counts of 2 or more were considered positive (Figure 2).

\section{Statistical analysis}

Data analysis was conducted using SPSS version 20.0 statistical software (IBM, Armonk, NY, USA). Numerical data were directly recorded, and categorical data were 


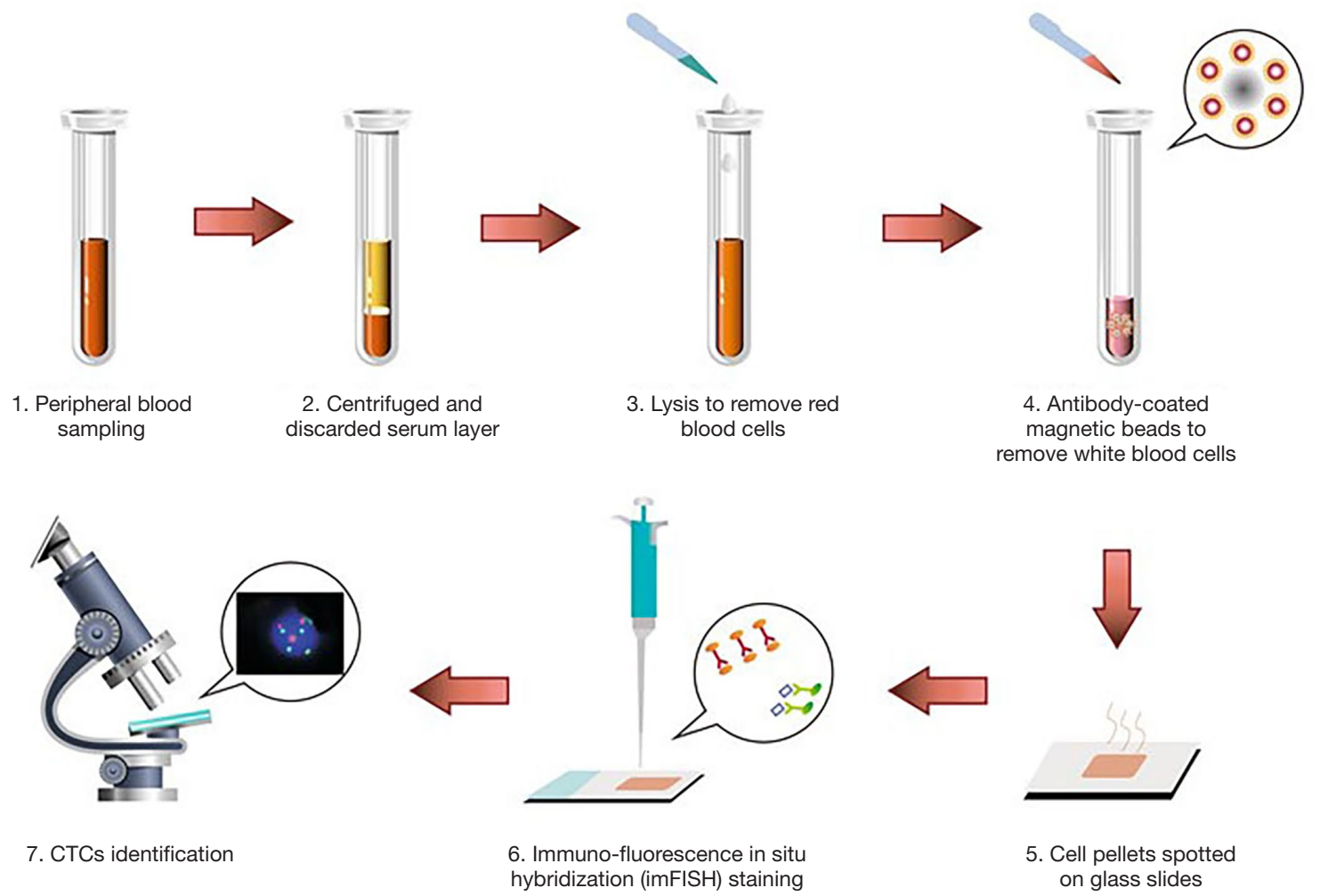

Figure 1 Flow chart of CTCs enrichment and identification. CTC, circulating tumor cell.

recorded in different categories. The Kaplan-Meier survival estimates were used for survival analysis, and the log rank test was used for comparing between strata. The correlation between level of CTCs and major clinicopathological characteristics was examined using Pearson's $\chi^{2}$ test. A twosided $\mathrm{P}<0.05$ was considered as statistically significant.

\section{Results}

\section{Major clinical-pathologic characteristics}

Seventy-three patients were enrolled in the study. Table 1 shows the details of major clinical-pathologic characteristics of these patients.

\section{Results of CTCs detection}

The median number of CTCs was 2 (range 0-9) in 73 patients. The positive rate of overall CTCs was $65.8 \%$ (48/73). That of recurrent patients versus no recurrent patients was $87.5 \%(14 / 16)$ versus $59.6 \%(34 / 57)$. The difference was statistically significant $(\mathrm{P}=0.038)$. Quantitatively, the mean number of CTCs was 3.69 in recurrence group and 2.46 in no recurrence group. Moreover, correlation analysis showed that the major clinicopathological parameters had no significant correlation with the level of post-operative CTCs, except for recurrence (Table 2).

\section{RFS analysis}

The median follow-up time was 22.1 (range 3.5-35.9) months. The mean RFS was 30.6 months [95\% confident interval (CI): 28.3-32.9 months] for all 73 patients. The mean RFS of CTC-positive and -negative group were 28.7 (95\% CI: 25.7-31.7) months and 34.0 (95\% CI: $31.5-$ 36.5) months $(\mathrm{P}=0.043)$ (Figure $3 A$ ).

According to CTCs detecting results and post-operative adjuvant chemotherapy, patients were divided into four subgroups, marked as CTC+CHEMO+, CTC-CHEMO+, 

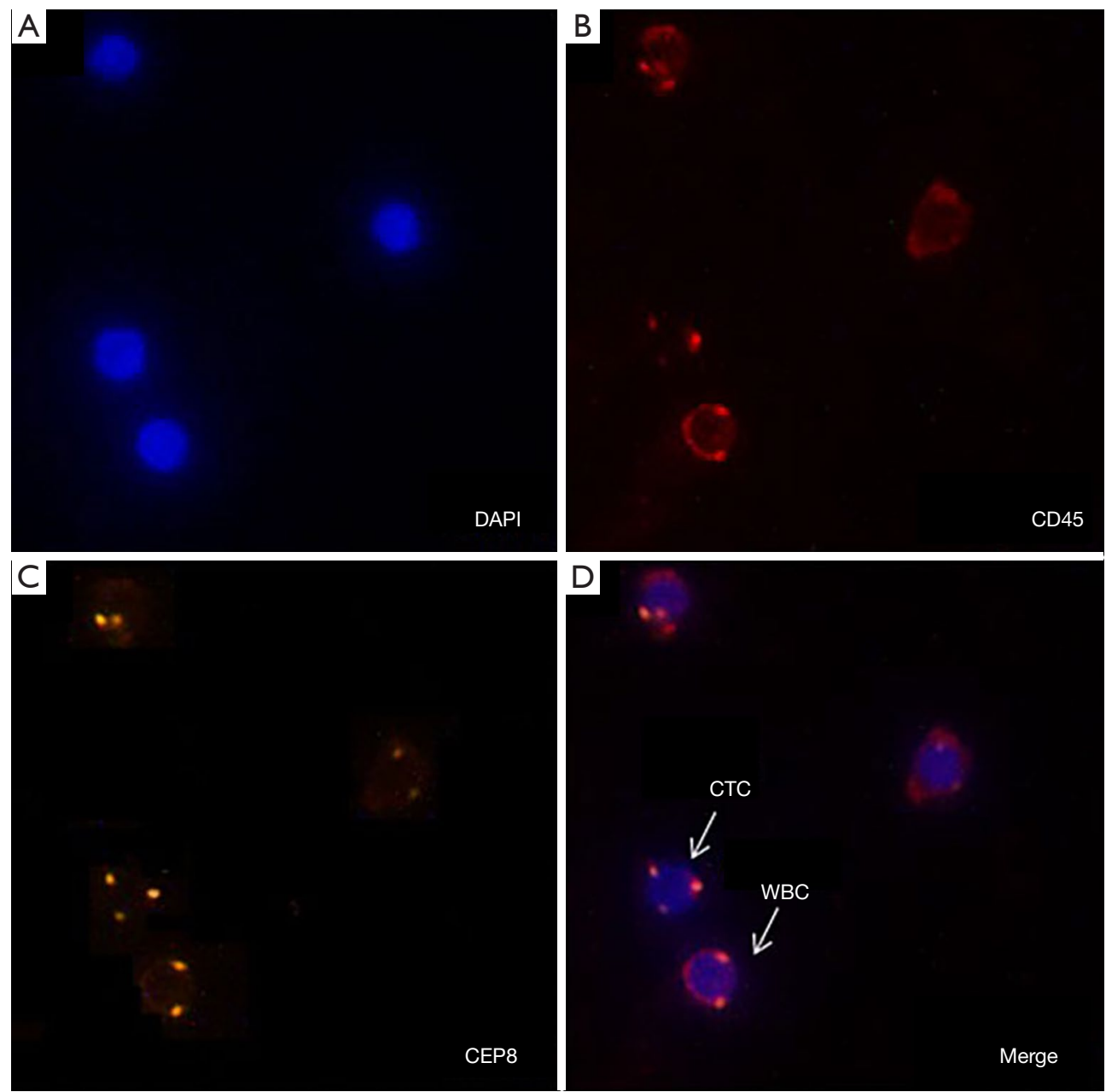

Figure 2 Identification of CTCs by imFISH staining. (A) DAPI: blue; (B) CD45: red; (C) CEP8: orange; (D) merged image. The cell was considered as a CTC, when it was CD45 negative and CEP8 with hyperdiploid. CTC, circulating tumor cell; imFISH, immunefluorescence in situ hybridization.

CTC+CHEMO-, and CTC-CHEMO-. The mean RFS of $\mathrm{CTC}+\mathrm{CHEMO}+$ and CTC-CHEMO+ were not reached. The mean RFS of CTC+CHEMO- and CTC-CHEMOwere 27.7 (95\% CI: 24.4-31.1) months and 33.4 (95\% CI: 30.3-36.5) months. Log rank test showed a P value of 0.053 (Figure 3B).

\section{Discussion}

Administration of adjuvant chemotherapy in major stage II CRC patients is regarded as "overtreatment", even though about $20 \%$ of these patients will suffer recurrence or metastasis within 5 years. This study found that stage II CRC patients with negative CTCs had a significant longer
RFS than those with positive CTCs. Moreover, adjuvant chemotherapy could erase this RFS difference. These results indicated that CTCs could be used as an effective prognostic marker for selecting stage II CRC patients for adjuvant chemotherapy.

It is not recommended for routine use of adjuvant chemotherapy in stage II CRC patients, due to that only a small subgroup of patients benefit from it, whereas for others, there is harm, a worse quality of life, and no net benefit $(8,9)$. However, it is considered reasonable for administration of adjuvant chemotherapy in selected patients with high risk disease, despite lacking direct evidence. As of now, no certain clinicopathologic characteristics or other factors have been identified as 
Table 1 Major clinicopathological characteristics of 73 patients

\begin{tabular}{|c|c|}
\hline Characteristics & Value, $n(\%)$ \\
\hline Median age (yr, range) & $64.3[29-85]$ \\
\hline \multicolumn{2}{|l|}{ Gender } \\
\hline Female & $40(54.8)$ \\
\hline Male & $33(45.2)$ \\
\hline \multicolumn{2}{|l|}{ Differentiation } \\
\hline Well differentiation adenocarcinoma & $3(4.1)$ \\
\hline Moderate differentiation adenocarcinoma & $66(90.4)$ \\
\hline Poor differentiation adenocarcinoma & $4(5.5)$ \\
\hline \multicolumn{2}{|l|}{ Primary tumor location } \\
\hline Ascending colon & $14(19.2)$ \\
\hline Transverse colon & $4(5.5)$ \\
\hline Descending colon & $6(8.2)$ \\
\hline Sigmoid colon & $29(39.7)$ \\
\hline Rectum & $20(27.4)$ \\
\hline \multicolumn{2}{|l|}{ Tumor type } \\
\hline Ulcerative & $54(74.0)$ \\
\hline Protrude & $19(26.0)$ \\
\hline \multicolumn{2}{|l|}{ Surgical approach } \\
\hline Laparoscopic & $43(58.9)$ \\
\hline Open & $21(28.8)$ \\
\hline Converted & 9 (12.3) \\
\hline \multicolumn{2}{|l|}{ Recurrence } \\
\hline No & $57(78.1)$ \\
\hline Yes & $16(21.9)$ \\
\hline \multicolumn{2}{|l|}{ Adjuvant chemotherapy } \\
\hline No & $59(80.8)$ \\
\hline Yes & $14(19.2)$ \\
\hline \multicolumn{2}{|l|}{ T stage } \\
\hline T3 & 67 (91.8) \\
\hline $\mathrm{T} 4$ & $6(8.2)$ \\
\hline \multicolumn{2}{|l|}{ Perivascular invasion } \\
\hline No & $54(74.0)$ \\
\hline Yes & $19(26.0)$ \\
\hline
\end{tabular}

predictive markers for adjuvant chemotherapy in stage II CRC patients. Literature reviews showed that T4 lesion, perforation, and poorly differentiated histology might be considered as candidates for adjuvant chemotherapy, but there were still no adequate evidence (9). In fact, we found that these three characteristics had no significant influence on RFS in stage II CRC patients. Moreover, patients with negative post-operative CTCs detection had a significant longer RFS than those with positive CTCs.

CTCs or tumor-derived biomarkers detection from biological fluids of cancer patients were recognized as "liquid biopsy" in solid tumors (10). Liquid biopsy has several advantages compared with tissue biopsy, such as low invasive procedure, fast turnaround time and the ability to provide a more comprehensive molecular portrait of the disease as compared with the analysis of a small fragment of a single tumor lesion (3). Liquid biopsy has been more and more commonly studied and used in the clinical management of CRC patients, involving early diagnosis and prognostic assessment in early CRC, identification of prognostic and predictive markers in advanced CRC, and monitoring response to therapy and clonal evolution of the disease (3).

CTCs test in early stage CRC relied more sensitive and specific detecting methods. Recently, detecting methods based on analyzing cell-free DNA were widely used in early stage CRC. Moreover, a new negative enrichment and imFISH technique was used to detect CTCs in a variety of cancers, such as colorectal cancer (CRC) (11-13), breast cancer $(13,14)$, lung cancer $(13,14)$ and prostate cancer $(13)$. The cut-off of two or more CTCs $/ 7.5 \mathrm{ml}$ was chosen to define as positive (11-14). This method had more effective enrichment, higher sensitivity and better specificity, compared to the traditional method based on epithelial cell adhesion molecule (EpCAM) enrichment. In addition, the timing of sampling was also important. Wang et al. reported that surgery affected the number of detected CTCs, and CTCs had no prognostic significance in the early postoperative until 7 days after surgery (15). Therefore, we chose to take peripheral blood samples on the 7 th postoperative day. Our study showed that the positive rate of CTCs was $65.8 \%$, and the level of CTCs was only significantly related to recurrence and had no correlation to other clinic-pathological characteristics. These results were not consistent with the results in metastatic CRCs (16), 
Table 2 Correlation between CTCs detection results and major clinicopathological characteristics

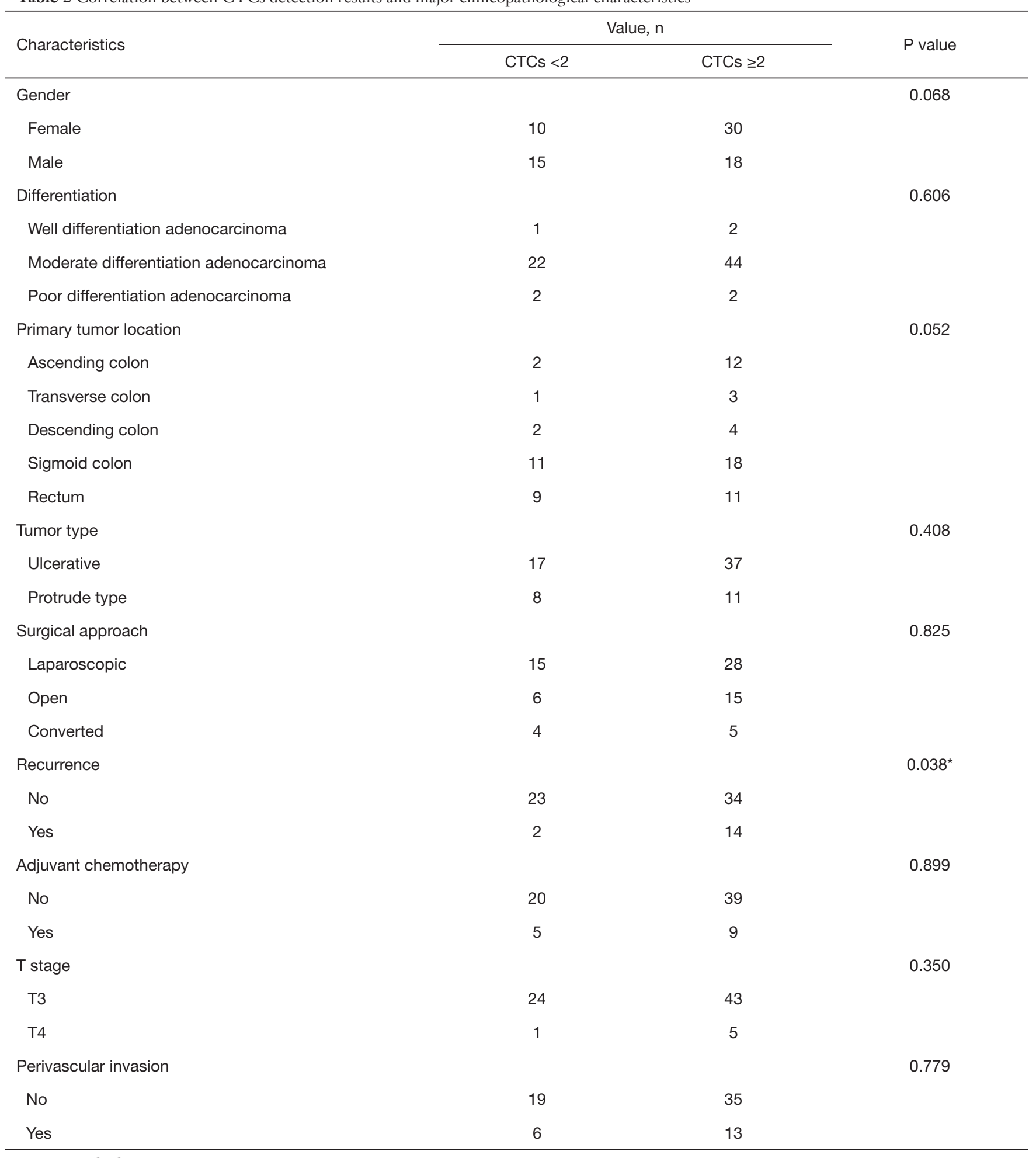

*, $\mathrm{P}<0.05$. CTC, circulating tumor cell. 

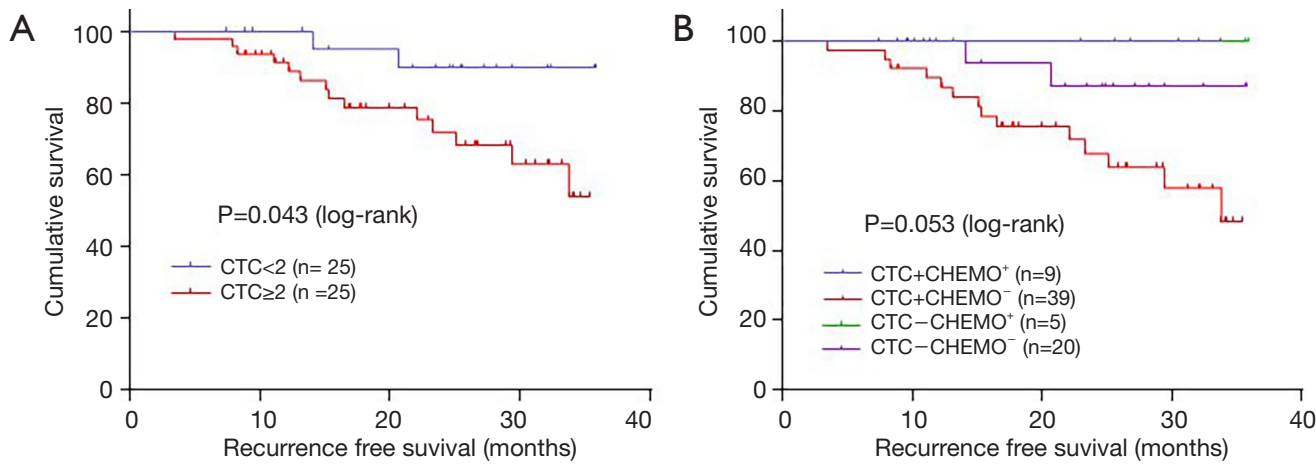

Figure 3 RFS analysis depending on post-operative CTCs (A) and both CTCs and adjuvant chemotherapy (B). RFS, recurrence free survival; CTC, circulating tumor cell.

suggesting that CTCs may be an independent prognostic factor in early stage II CRC patients. However, we still need more direct evidence to confirm the utility of CTCs in early stage CRC.

According to published literatures, the recurrent rate of stage II CRC patients received radical treatment ranged from $10 \%$ to $30 \%(17,18)$. In this study, the recurrence rate was $21.9 \%(16 / 73)$, which was similar to the previous results. The level of CTCs also showed significant difference between patients experienced recurrence and those with no recurrence. Moreover, the patients with positive CTCs had a significant shorter RFS than the negative ones. As QUASAR trial showed, adjuvant chemotherapy could improve survival of patients with stage II CRC (19). In our study, 9 of 48 CTC-positive patients received adjuvant chemotherapy and had no recurrence. The remaining 39 patients with positive CTCs did not receive chemotherapy, and 14 patients had recurrence. The difference was nearly significant with a $\mathrm{P}$ value of 0.053 , it might be interpreted as the insufficient follow-up and few recurrence events.

The present study had some limitations. Firstly, the sample size of this study was small. Secondly, the followup time was short, and the positive events were not enough. We had to replace median RFS with mean RFS, which reduced the power of evidence. Moreover, we failed to perform multivariate analyze due to too many censored data. Therefore, these findings in this study required more confirmations from higher levels of evidence. Nonetheless, these potential limitations do not invalidate our findings that are descriptions of the important utility of CTCs.

In conclusion, our study indicates that the level of CTCs could be used as a prognostic factor to predict RFS in stage
II CRC patients, and has utility as a biomarker for the selection of patients for adjuvant chemotherapy.

\section{Acknowledgments}

Funding: This study was supported by Beijing Municipal Administration of Hospitals Clinical Medicine Development of Special Funding Support (ZYLX201504), National Key Technologies R\&D Program (2015BAI13B09) and Beijing Health System of High Level Health Technical Personnel Training Project (2015-3-005).

\section{Footnote}

Conflicts of Interest: All authors have completed the ICMJE uniform disclosure form (available at http://dx.doi. org/10.21037/tcr.2020.01.37). The authors have no conflicts of interest to declare.

Ethical Statement: The authors are accountable for all aspects of the work in ensuring that questions related to the accuracy or integrity of any part of the work are appropriately investigated and resolved. The study was conducted in accordance with the Declaration of Helsinki (as revised in 2013). The study design was approved by the Ethical Committee of the Beijing Friendship Hospital (Ethics number: BJFH-EC/2014-086) and informed consent was taken from all individual participants.

Open Access Statement: This is an Open Access article distributed in accordance with the Creative Commons Attribution-NonCommercial-NoDerivs 4.0 International License (CC BY-NC-ND 4.0), which permits the non- 
commercial replication and distribution of the article with the strict proviso that no changes or edits are made and the original work is properly cited (including links to both the formal publication through the relevant DOI and the license). See: https://creativecommons.org/licenses/by-nc-nd/4.0/.

\section{References}

1. Chen $W$, Zheng R, Baade PD, et al. Cancer statistics in China, 2015. CA Cancer J Clin 2016;66:115-32.

2. Boland CR, Goel A. Prognostic Subgroups among Patients with Stage II Colon Cancer. N Engl J Med 2016;374:277-8.

3. Normanno N, Cervantes A, Ciardiello F, The liquid biopsy in the management of colorectal cancer patients: Current applications and future scenarios. Cancer Treat Rev 2018;70:1-8.

4. Meyers BM, Cosby R, Quereshy F, Adjuvant Chemotherapy for Stage II and III Colon Cancer Following Complete Resection: A Cancer Care Ontario Systematic Review. Clin Oncol (R Coll Radiol) 2017;29:459-65.

5. Rothé F, Maetens M, Rouas G, et al. CTCs as a prognostic and predictive biomarker for stage II/III Colon Cancer: a companion study to the PePiTA trial. BMC Cancer 2019;19:304.

6. Sun Q, Liu Y, Liu B, Use of Liquid Biopsy in Monitoring Colorectal Cancer Progression Shows Strong Clinical Correlation. Am J Med Sci 2018;355:220-7.

7. Provenzale D, Gupta S, Ahnen DJ, et al. NCCN Guidelines Insights: Colorectal Cancer Screening, Version 1.2018. J Natl Compr Canc Netw 2018;16:939-49.

8. Lewis C, Xun P, He K. Effects of adjuvant chemotherapy on recurrence, survival, and quality of life in stage II colon cancer patients: a 24-month follow-up. Support Care Cancer 2016;24:1463-71.

9. Takenaka Y, Miyoshi N, Fujino S, et al. Development of a novel prediction model for recurrent stage II colon cancer. Surg Today 2019. [Epub ahead of print].

Cite this article as: Yu JH, Wang D, Jin L, Wang J, Zhao XM, Wu GC, Yao HW, Yang YC, Zhang ZT. Utility of circulating tumor cells in stage II colorectal cancer patients undergoing curative resection. Transl Cancer Res 2020;9(3):1487-1494. doi: $10.21037 /$ tcr.2020.01.37
10. Lianidou ES, Markou A, Strati A. The Role of CTCs as Tumor Biomarkers. Adv Exp Med Biol 2015;867:341-67.

11. Nanduri LK, Hissa B, Weitz J, et al. The prognostic role of circulating tumor cells in colorectal cancer. Expert Rev Anticancer Ther 2019;19:1077-88.

12. Kassam Z, Burgers K, Walsh JC, et al. A prospective feasibility study evaluating the role of multimodality imaging and liquid biopsy for response assessment in locally advanced rectal carcinoma. Abdom Radiol (NY) 2019;44:3641-51.

13. Bielčiková $Z$, Jakabová $A$, Pinkas $M$, et al. Circulating tumor cells: what we know, what do we want to know about them and are they ready to be used in clinics? Am J Transl Res 2017;9:2807-23.

14. Li Y, Ma G, Zhao P, et al. Improvement of sensitive and specific detection of circulating tumor cells using negative enrichment and immunostaining-FISH. Clin Chim Acta 2018;485:95-102.

15. Wang L, Zhou S, Zhang W, et al. Circulating tumor cells as an independent prognostic factor in advanced colorectal cancer: a retrospective study in 121 patients. Int J Colorectal Dis 2019;34:589-97.

16. Tan K, Leong SM, Kee Z, et al. Longitudinal monitoring reveals dynamic changes in circulating tumor cells (CTCs) and CTC-associated miRNAs in response to chemotherapy in metastatic colorectal cancer patients. Cancer Lett 2018;423:1-8.

17. Graf W, Cashin PH, Ghanipour L, et al. Prognostic Impact of BRAF and KRAS Mutation in Patients with Colorectal and Appendiceal Peritoneal Metastases Scheduled for CRS and HIPEC. Ann Surg Oncol 2020;27:293-300.

18. Tsikitis VL, Larson DW, Huebner M, et al. Predictors of recurrence free survival for patients with stage II and III colon cancer. BMC Cancer 2014;14:336.

19. Quasar Collaborative Group, Gray R, Barnwell J, et al. Adjuvant chemotherapy versus observation in patients with colorectal cancer: a randomised study. Lancet 2007;370:2020-9. 